\title{
BMJ Open First incidence, age of onset outcomes and risk factors of onset of DSM-5 oppositional defiant disorder: a cohort study of Spanish children from ages 3 to 9
}

\author{
Lourdes Ezpeleta, ${ }^{1} \mathrm{~J}$ Blas Navarro, ${ }^{2}$ Nuria de la Osa, ${ }^{1}$ Eva Penelo, ${ }^{2}$ \\ Josep Maria Domènech ${ }^{2}$
}

To cite: Ezpeleta L, Navarro JB, de la Osa N, et al. First incidence, age of onset outcomes and risk factors of onset of DSM-5 oppositional defiant disorder: a cohort study of Spanish children from ages 3 to 9 . BMJ Open 2019;9:e022493. doi:10.1136/ bmjopen-2018-022493

- Prepublication history and additional material for this paper are available online. To view these files, please visit the journal online (http://dx.doi org/10.1136/bmjopen-2018022493).

Received 22 February 2018 Revised 22 January 2019 Accepted 11 February 2019

Check for updates

(C) Author(s) (or their employer(s)) 2019. Re-use permitted under CC BY-NC. No commercial re-use. See rights and permissions. Published by BMJ.

${ }^{1}$ Unitat d'Epidemiologia i de Diagnòstic en Psicopatologia del Desenvolupament, Departament de Psicologia Clínica i de la Salut, Universitat Autònoma de Barcelona, Bellaterra, Barcelona, Catalunya, Spain

2Unitat d'Epidemiologia i de Diagnòstic en Psicopatologia del Desenvolupament, Departament de Psicobiologia i Metodologia de les Ciències de la Salut, Universitat Autònoma de Barcelona, Bellaterra, Barcelona, Catalunya, Spain

Correspondence to

Dr Lourdes Ezpeleta;

Lourdes.Ezpeleta@uab.cat

\section{ABSTRACT}

Objective To examine the 1-year first incidence and prevalence of oppositional defiant disorder (ODD), the outcomes on psychopathology and functioning by age of onset and the risk factors of onset of ODD from ages 3 to 9 in children from the Spanish general population.

Design Longitudinal with seven follow-ups and double cohort (ODD and non-ODD children).

Setting General population of preschool and elementary school children in Barcelona (Spain).

Participants On a first phase, the parent-rated Strengths and Difficulties Questionnaire conduct problems scale plus ODD Diagnostic and Statistical Manual of Mental Disorders, fourth version, symptoms were used to screen for behavioural problems. The second phase sample size contained 622 cases at age 3 and, at age 9, 418 remained in the study.

Results The probability of the onset of ODD showed increasing values at ages $4(\mathrm{R}=2.7 \%)$ and 5 years $(R=4.4 \%)$. These values decreased until age $7(R=1.9 \%)$ and increased again until age $9(\mathrm{R}=3.6 \%)$. Up to 9 years old, the cumulative risk of new cases of ODD was $21.9 \%$. Early onset was associated with a higher risk of depression comorbidity and later onset with higher functional impairment and symptomatology. Subthreshold ODD, high scores in irritability and headstrong dimensions, attention deficit/hyperactivity disorder and other comorbidity, negative affectivity until age 7 , difficulties in inhibit and emotional control, punitive parenting and maternal internalising problems were risk factors of a first episode of ODD during this 7-year period.

Conclusions The risk of new cases of ODD in the general population at preschool age and during childhood is high. Preschool age is a target period for preventive interventions. Identified risk factors are objectives for targeted and indicated interventions.

\section{INTRODUCTION}

According to epidemiological studies, the proportion of children and adolescents with mental health problems is $13.4 \%$. $^{1}$ These disorders are stable and continue into later life with adverse adults outcomes. ${ }^{2}$ Therefore, childhood is a target period for the
Strengths and limitations of the study

The length of the follow-up period (7 years) including two different developmental stages, preschool and childhood.

- The information on risk factors and outcomes obtained from parents and teachers.

- The consideration of risk factors in Cox regression models as time-dependent covariates instead of fixed covariates.

- The lower participation of low socioeconomic status families and the non-random attrition for some outcomes may have led to bias in the estimates.

- The low incidence of oppositional defiant disorder (ODD) made necessary to cluster ages ( $3-5$ and 6-9) for the analysis of the influence of ODD age of onset on psychopathology and functioning.

early identification and prevention of mental disorders.

Oppositional defiant disorder (ODD), a pattern of negativistic, defiant, disobedient and hostile behaviour, is one of the most prevalent disorders from preschool age to adulthood. ${ }^{34}$ The pooled prevalence is $3.6 \%$ up to age $18 .{ }^{1}$ ODD is accompanied by various concurrent disorders (attention deficit/ hyperactivity disorder $[\mathrm{ADHD}])$, successive comorbidity (conduct disorder, anxiety, depression, substance use $)^{56}$ and functional impairment. ${ }^{7} 8$ Symptomatology is stable and sufferers have difficulties in the transition to adulthood. ${ }^{9}$ The amount of children and families affected and the severe consequences that compromise healthy mental development underscore the need to know when the first onset occurs and the factors that predict this onset in order to plan appropriate preventive strategies.

Currently, we know how many children in the population are affected by ODD at a given point in time, that is, the prevalence, 
a measure of the status of the disease. We do not know, however, how many new cases appear at different developmental stages, that is, incidence, a measure of newly occurring cases of the disease during a specific developmental period. ${ }^{10}$ Because there is often a low number of incident cases, incidence studies require cohort designs with large size samples. Literature shows that there is a dearth of studies about the incidence of psychiatric disorders in childhood and adolescence. The available data on ODD mostly focus on adolescents and youths. Roberts ${ }^{11}$ reported that the risk of new cases of ODD for adolescents in a 12-month period was $1.56 \%$, and Benjet ${ }^{12}$ found a $5 \%$ 8-year incidence for 19 - to 26 -year-old youth. There are no studies on the incidence of ODD during preschool and childhood. Neither do we know the differential consequences of the disorder according to age of onset. Literature on general mental disorders has reported that early onset is associated with greater severity, persistence and lack of response to treatment. ${ }^{13}$ Age of onset is an important data to advise on mental health policies. ${ }^{14}$

Several risk factors have been reported in the literature on ODD. Child risk factors include genetic influences, ${ }^{15}$ difficult temperament, ${ }^{16}$ difficulties in processing social information, ${ }^{17} \operatorname{sex}^{18}$ and ADHD. ${ }^{19}$ The contextual factors reported include socioeconomic status (SES), parenting practices, parental psychopathology, family conflict and poor attachment. ${ }^{15} 20$ Incidence figures, which report on new cases of disease, are more useful for identifying risk factors than prevalence studies, which include both chronic and new cases. ${ }^{21}$ No previous studies have examined the risk factors of ODD by considering new cases. Only Roberts ${ }^{11}$ adopted this approach in adolescents, reporting that a younger age, poor family satisfaction, passive coping and low mastery, school and economic stress and poor relations with parents were predictors of incident cases of ODD.

Furthermore, ODD is a continuous disorder that starts early in life and persists into adulthood. ${ }^{9}$ It is therefore imperative to know for prevention purposes how the early manifestations of ODD symptomatology affect the definite appearance of the full disorder. Several dimensions of ODD have been identified to explain its underlying structure: irritable (including loses temper, angry and touchy); headstrong (argues, defies, annoys, blames) and hurtful (spiteful-vindictive) ${ }^{22}$ Rowe $^{20}$ showed how ODD dim ensions predict full ODD diagnosis. Moreover, the literature has shown that subthreshold conditions are risk factors for developing similar (homotypic) or different (heterotypic) full syndrome ${ }^{23}$ and that they constitute a major public mental health burden. ${ }^{24}$

The objective was to study annually the proportion of incident cases of ODD from ages 3 to 9 (preschool through childhood), to ascertain the differential outcomes by age of onset and to test if previously reported risk factors associated with ODD are prospective risk factors of incident cases at these developmental stages.

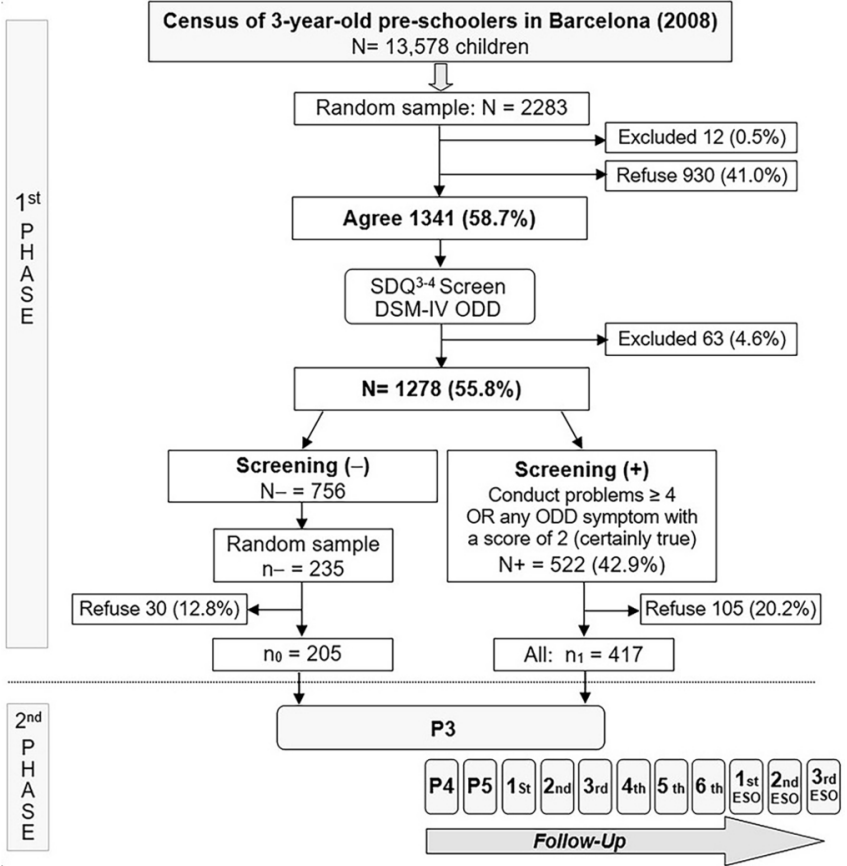

Figure 1 Design of the study. DSM-IV, Diagnostic and Statistical Manual of Mental Disorders, fourth version; ODD, oppositional defiant disorder; SDQ, Strengths and Difficulties Questionnaire.

\section{METHOD}

\section{Participants}

The initial sample consisted of 2283 children randomly selected from early childhood schools in Barcelona (Spain). ${ }^{25} \mathrm{~A}$ two-phase design was employed. In the first phase of sampling, 1341 families $(58.7 \%)$ agreed to participate $(33.6 \%$ high SES, $43.1 \%$ middle and $23.3 \%$ low; $50.9 \%$ boys). To ensure that children with possible behavioural problems participated, the parent-rated Strengths and Difficulties Questionnaire $\left(\mathrm{SDQ}^{3-4}\right)$ conduct problems scale ${ }^{26}$ plus ODD Diagnostic and Statistical Manual of Mental Disorders, fourth version (DSM-IV) symptoms were used to screen. Two groups were considered: screen positive (all children with SDQ scores $\geq 4$, percentile 90 , or with a positive response to any of the eight DSM-IV ODD symptoms) and screen-negative (a random group comprising $28 \%$ of children who did not reach the positive threshold). The sample size was determined for detecting OR $\geq 1.8$ between psychopathology and risk factors, using a test of hypothesis for risk $\alpha=0.05$ and power of 0.80 . As the planned follow-up was 12 years long, the sample size was increased $50 \%$ for losses.

The final sample for the follow-ups (second phase) included 622 children (figure 1) comprising all the children from the screen-positive group whose families accepted to participate $(\mathrm{n}=417 ; 49.4 \%$ boys $)$ and a random sample from the screen-negative group $(n=205$; $51.2 \%$ boys). To select participants from screen-negative group, children of each classroom were alphabetically numbered without including the name of the child nor the school. Then they were randomly permutated 
Table 1 Demographic characteristics of the sample at age $3(n=622)$

\begin{tabular}{lc}
\hline Age (mean; SD) & $3.8(0.33)$ \\
\hline Sex; $n$ (\%) & $311(50.0)$ \\
Male & \\
\hline Race/ethnicity; $n$ (\%) & $557(89.5)$ \\
\hline Non-Hispanic White & $46(7.4)$ \\
Hispanic-American & $19(3.1)$ \\
Other & \\
Socioeconomic status; $n$ (\%) & $205(33.0)$ \\
\hline High & $280(45.0)$ \\
Middle & $137(22.0)$ \\
\hline Low & \\
\hline
\end{tabular}

using SPSS random number generator, and the first $30 \%$ was selected. The percentage of dropouts at annual follow-up from ages 4 to 9 was similar in the two screen groups $\left(\chi^{2}=0.72, \mathrm{p}=0.798\right.$ at age $4 ; \chi^{2}=0.31, \mathrm{p}=0.575$ at age $5 ; \chi^{2}=1.36, \mathrm{p}=0.244$ at age $6 ; \chi^{2}=0.02, \mathrm{p}=0.877$ at age $7 ; \chi^{2}=0.49$ and $\mathrm{p}=0.484$ at age $8 ; \chi^{2}=0.20$ and $\mathrm{p}=0.652$ at age 9). No differences in sex $\left(\chi^{2}=0.07 ; \mathrm{p}=0.793\right)$ or type of school $\left(\chi^{2}=0.72 ; p=0.396\right)$ were found on comparing completers and dropouts during the 7 years of annual follow-ups. However, the SES of those leaving the study until age 9 was lower $\left(\chi^{2}=20.89 ; \mathrm{p}<0.001\right)$. Finally, to assess randomness of attrition, the outcome scores at age 3 between cases and dropouts at age 9 were compared. For 6 out of the 16 outcomes, scores at age 3 were higher for dropouts than for completers at age 9 .

From the initial 622 children, 65 who presented an ODD diagnosis at the start of the study (age 3) and 18 who left the study at the second follow-up (age 4) were excluded for the analysis of risk factors because of lack of information $(n=539)$. Decrements in sample size at successive follow-ups were either due to attrition or to the exclusion of children who had already presented a first ODD diagnosis. Demographic characteristics are shown in table 1.

\section{Measures}

Diagnostic interview of children and adolescents for parents of preschool children

The diagnostic interview of children and adolescents for parents of preschool children ${ }^{27}$ is a computerised semistructured interview which generates diagnoses through algorithms following DSM-V. The diagnosis of ODD was obtained annually. The interviews in the first assessment gathered data from the first 3 years of life. ADHD, major depression, any anxiety disorders (separation, generalised, social anxiety or specific phobias) and comorbidity (ADHD, conduct disorder, major depression or any anxiety plus ODD) were obtained at each age from 3 to 9 years old. Subthreshold ODD was defined as cases that did not meet the threshold criteria of four symptoms for the diagnosis but presented impairment or distress.
Rowe's ${ }^{20}$ ODD dimensions were used (irritable and headstrong). Interinterviewer agreement in the diagnoses ranged from kappa coefficients from 0.83 to 1 (mean kappa 0.92; being 0.84 for ODD), indicating a good to excellent agreement between interviewers.

$\mathrm{SDQ}^{26}$ assesses emotional and behavioural problems with 25 items with three response options organised in five scales. It was answered by the parents and teachers. Cronbach's alpha for parents range from 0.55 (conduct) to 0.85 (hyperactivity) and for teachers from 0.69 (conduct) to 0.88 (total).

The Children's Global Assessment Scale ${ }^{28}$ is a global measure of functional impairment rated by the interviewer. Scale scores range from 1 (maximum impairment) to 100 (normal functioning). Scores above 70 indicate normal adaptation.

Children's Behaviour Questionnaire Short Form and Very Short Form ${ }^{29}$ measure reactive and self-regulative temperament with 94 and 36 items, respectively, on a seven-point Likert-type scale. These were answered by the parents when the children were 3,4 and 5 years old (short form) and 7 years old (very short form). The broad dimensions negative affectivity and effortful control were considered. Cronbach's alpha in the sample ranged from 0.71 for effortful control at age 7 to 0.85 for negative affectivity at age 5 .

The Behaviour Rating Inventory of Executive Function preschool version, ${ }^{30}$ answered by teachers when children were 3 years old, assesses behaviours reflecting the executive functions in daily life. The broad dimension that combine inhibit (control of impulses and behaviour) and emotional control (appropriate modulation of emotional responses) (Inhibitory Self-Control Index; ISCI) was used (Cronbach's alpha: 0.94).

The Alabama Parenting Questionnaire-Preschool $(\mathrm{APQ}-\mathrm{Pr})^{31}$ measures parental practices in three dimensions (24 items): positive discipline techniques, inconsistent parenting and punitive parenting. ${ }^{32}$ They were obtained at ages 3 and 6 . Cronbach's alpha for the three dimensions was $0.75,0.62$ and 0.42 at age 3 and $0.74,0.66$ and 0.52 at age 6 , respectively.

The Adult Self-Report ${ }^{33}$ assesses dimensional psychopathology (126 items) in adults. The mothers answered the questionnaire when the children were 3 and 8 years old. Internalising and externalising scale scores were analysed (Cronbach's alpha 0.85 and 0.80 , respectively, at the last follow-up).

\section{Patient and public involvement statement}

ODD is a social problem and families and schools complain about how to manage disruptive behaviour disorders at home and in school. We wanted to investigate about the development of this problem to know the best developmental moments and their risk factors to help the families and the teachers to prevent oppositionality. Families and schools were freely and actively involved in the study. Families and schools were informed yearly of the results of the previous follow-up and were oriented 
about what to do to improve the behaviour when necessary. Every 3 years, they received a written report about the evolution and development of the child. Teachers received a 15-hour course about How to manage disruptive behaviour disorder at the school-room at the beginning of different school levels (preschool: age 3, elementary:ages 6 and 9$)$.

\section{Procedure}

Families were recruited in schools and gave written consent. The families who agreed to participate and met the screening criteria were contacted each year and interviewed in school. Interviewers were trained and were blind to the screening group. All the interviews were audiorecorded and supervised. The data were collected between November 2009 and July 2016.

\section{Statistical analysis}

The data were analysed with Stata V.15 for Windows. Since all the data were collected using a double-phase screening design, all analyses were weighted by assigning each child a value that was inverse to the probability of random selection in the second phase of sampling. Cases with missing data were excluded separately for each analysis (pairwise deletion). The incidence proportion was calculated for 1 year time periods beginning at 4 years old by dividing the number of new cases of first ODD diagnosis (incident cases) by the number of children at risk, that is, the number of cases at the beginning of the period excluding those who had previous diagnoses of ODD. This ratio is also called Risk (R) and it estimates the "probability of an event during a specified period of time' $\cdot{ }^{10}$ Cumulative risk estimates the risk of ODD from 0 years old to each time period; because of the lost cases across the study, cumulative risk was computed by the Kaplan-Meier product-limit estimation ${ }^{34}$ using the weighted annual risk.

The analysis of differences in psychopathology and functioning by age of onset of ODD was made by analysis of variance for raw scores of quantitative outcomes and logistic regression for binary outcomes. Age of onset was grouped into preschool (ages 3-5) and school (ages $6-9)$ periods. The group without ODD was also considered and post hoc comparisons corrected by Bonferroni for multiple comparisons were estimated. Treatment for ODD at any time, current ODD diagnosis and number of years with an ODD diagnosis were introduced as covariates to adjust for confounding effects.

To analyse the predictors of the risk of an ODD diagnosis, several Cox proportional hazard regression models were estimated, grouping predictors (risk factors) by the measurement instrument and adjusting estimates by sex and SES. Predictors were considered as time dependent between ages 3 and 8 to benefit from the most recent available information. As a consequence and because of the multiple-record structure of the data matrix (each child had one data record for each follow-up period), the robust variance estimator ${ }^{35}$ was used. No competitive events were considered due to the high specificity

\begin{tabular}{|c|c|c|c|}
\hline Age (years) & Total cases & ODD cases & 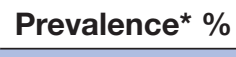 \\
\hline 3 & 622 & 65 & 6.03 \\
\hline 4 & 604 & 63 & 7.08 \\
\hline 5 & 535 & 46 & 7.09 \\
\hline 6 & 509 & 47 & 7.04 \\
\hline 7 & 456 & 41 & 6.99 \\
\hline 8 & 469 & 35 & 6.09 \\
\hline 9 & 418 & 40 & 8.83 \\
\hline
\end{tabular}

*Weighted by screen-positive or screen-negative membership: number of children with ODD divided by the total sample size at that age.

DSM-V, Diagnostic and Statistical Manual of Mental Disorders, fifth version; ODD, oppositional defiant disorder.

of an ODD diagnosis and to the characteristics of the sample, with neither deaths nor physical comorbidities that prevented an ODD diagnosis. Proportional hazard assumption was verified by calculating the significance value of the interaction between predictors and time. In the presence of significant interaction, the HR for the involved predictor was obtained separately for each year of follow-up, corresponding to ages 3 to 8 . For each Cox regression model, Harrell's C index ${ }^{36}$ was calculated to evaluate the adequacy of the predictions (values $\geq 0.70$ are considered good).

\section{RESULTS}

\section{Prevalence of ODD from 3 to 9 years old}

Table 2 presents the number of children in the study, the number of ODD diagnoses and the prevalence for each age. Prevalence oscillated between $6.0 \%$ (age 3) and $8.8 \%$ (age 9 ).

\section{First incidence and cumulative risk of ODD from 4 to 9 years old}

The first three columns in table 3 show the cases at risk (without an ODD diagnosis) at the beginning of each year period, the number of new cases diagnosed with ODD during that year and the incidence. The probability of the appearance of ODD showed a cubic shape, with risk increasing from age 4 to age $5(\mathrm{R}=2.7 \%$ to $4.4 \%)$, followed by a decrease until age $7(\mathrm{R}=1.9 \%)$ and a new increase at ages 8 and $9(\mathrm{R}=2.9 \%, 3.6 \%)$. The last column in table 3 shows the cumulative risk of having a first ODD diagnosis up to 9 years old, which reached $21.9 \%$. Figure 2 shows prevalence of ODD and incidence of first ODD diagnosis by age.

\section{Outcomes of age of onset of ODD}

Table 4 shows the scores and percentages of psychopathology and functioning for children with onset of ODD at 3-5 and 6-9 years old and for those without ODD, as well as the multiple comparisons between the three groups with the mean difference (MD) or the OR and 
Table 3 ODD 1-year first incidence and cumulative risk from 0 to 9 years old

\begin{tabular}{lllll}
\hline $\begin{array}{l}\text { Age } \\
\text { (years) }\end{array}$ & $\begin{array}{l}\text { Cases at } \\
\text { risk }\end{array}$ & $\begin{array}{l}\text { Incident } \\
\text { ODD } \\
\text { cases }^{*}\end{array}$ & \begin{tabular}{l} 
First ODD diagnosis \\
\cline { 4 - 4 }$\%$
\end{tabular} & $\begin{array}{l}\text { Risk } \\
\text { Cumulative } \\
\text { risk } \neq(\%)\end{array}$ \\
\hline 0 to 3 & & & 2.71 & 6.0 \\
\hline 4 & 541 & 23 & 4.39 & 12.6 \\
\hline 5 & 463 & 20 & 2.65 & 14.9 \\
\hline 6 & 419 & 13 & 1.88 & 16.5 \\
\hline 7 & 367 & 10 & 2.92 & 18.9 \\
\hline 8 & 373 & 11 & 3.61 & 21.9 \\
\hline 9 & 325 & 13 & \\
\hline
\end{tabular}

*Incident cases (after excluding children with previous diagnoses of ODD).

†Weighted by screen-positive or screen-negative membership. ‡Computed by Kaplan-Meier product-limit estimation using weighted annual risk.

ODD, oppositional defiant disorder.

their 95\% CI. Controlling by current ODD diagnosis, the number of years of duration of ODD and treatment received, children with onset at 3-5 years old scored lower on functional impairment, which indicates worse functioning ( $\mathrm{MD}=-7.17)$, and presented higher comorbidity with major depression $(\mathrm{OR}=5.76)$ in comparison to children without ODD. Children with onset of ODD at 6-9 years old scored higher on all the scales of parent's SDQ except prosocial (MD between 0.63 for conduct and 1.68 for hyperactivity) and on total (MD=3.95), and presented worse functioning $(\mathrm{MD}=-13.06)$ in comparison with children without ODD. There were differences in the total $\mathrm{SDQ}$ score $(\mathrm{MD}=2.99)$ and in peer problems $(\mathrm{MD}=0.66)$ between preschooler and late ODD onset, the latter showing higher scores. Moreover, children with onset of ODD at 6-9 years old presented higher functional impairment than those with onset at $3-5$ years old $(\mathrm{MD}=-5.89)$.

\section{Risk factors of incident ODD diagnosis from 3 to 9 years old}

HR for each risk factor with the $95 \% \mathrm{CI}$, its $\mathrm{p}$ value and Harrell's C for each model were calculated (see online supplementary table 1). The hazard of having an ODD diagnosis was increased by subthreshold ODD symptomatology $(\mathrm{HR}=6.27,95 \%$ CI 3.85 to 10.21), high scores on

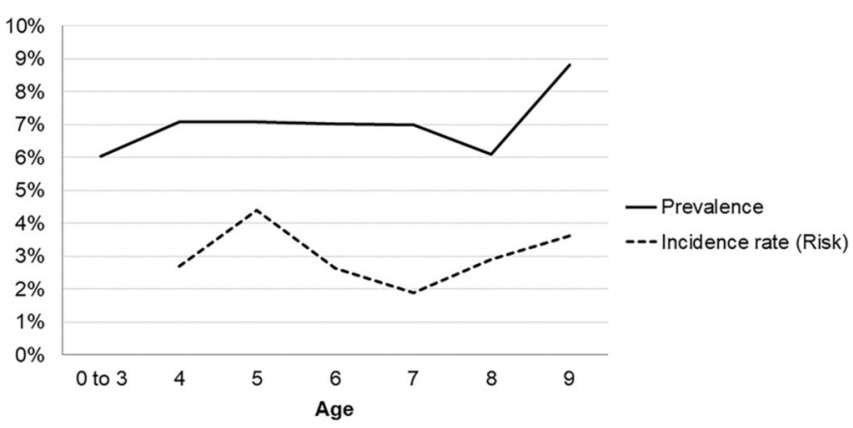

Figure 2 Prevalence and first incidence (risk) of oppositional defiant disorder diagnosed from 3 to 9 years old.
ODD dimensions of irritability (HR=1.56, 95\% CI 1.13 to 2.12) and headstrong (HR=2.33, 95\% CI 1.84 to 2.96), comorbidity ( $\mathrm{HR}=2.21,95 \% \mathrm{CI} 1.13$ to 3.53$)$, specifically of $\mathrm{ADHD}$ ( $\mathrm{HR}=2.64,95 \% \mathrm{CI} 1.42$ to 4.93 ), higher negative affectivity (HR=3.73, 95\% CI 2.21 to 6.29 at age three to $\mathrm{HR}=1.68,95 \%$ CI 1.27 to 2.23 at age 7 ), difficulties in inhibition and emotional control (HR=1.04, 95\% CI 1.02 to 1.07), higher scores in punitive parenting ( $\mathrm{HR}=1.22$, 95\% CI 1.08 to 1.38 ) and mother's internalising problems $(\mathrm{HR}=1.06,95 \%$ CI 1.02 to 1.10$)$.

The capability to predict new ODD first-incident cases from the subsets of risk factors was low in general. Only the first model with 'being an ODD subthreshold' as predictor, and the second model with 'ODD Irritability and Headstrong' scores as predictors showed Harrell's $\mathrm{C} \geq 0.70$.

\section{DISCUSSION}

To our knowledge, this is the first study reporting on not only the 1-year incidence of ODD in a 7-year follow-up design covering ages 3 to 9 and the effects of different age ranges of onset, but also their risk factors and the DSM-V prevalence. We found that the probability of the appearance of ODD shows a cubic shape with higher values for the preschool period, a decrease at the start of childhood (ages 6 and 7) and another increase when approaching puberty (ages 8 and 9). Prevalence was around 6\%-7\% between ages 3 and 8 , increasing to $8.8 \%$ by age 9 . An early onset of ODD is more closely associated with the presence of depressive comorbidity, but the functional impairment of those with later onset is most marked and their parents report higher symptomatology. Risk factors of incidence were identified.

Throughout development, prevalence was high and very stable $(6 \%-7 \%)$, with the highest value at 9 years old. These percentages indicate the need to allocate resources such as services and training to the parents, teachers and professionals involved with the children in these age ranges that have already developed the disorder.

2.7 and 4.4 out of 100 preschoolers aged 4 and 5, respectively, and between 1.9 and 3.6 out of 100 children aged 6 to 9 will develop a first episode of ODD in 1 year. It is remarkable that at the end of the follow-ups, the cumulative risk was high, indicating that up to 9 years old, the risk of presenting ODD is $21.9 \%$. This risk is highest in the preschool period, which cumulates $12.6 \%$ of the risk, the remaining $9.3 \%$ occurring in childhood. These values are noteworthy in terms of public mental health indicators if one considers the short-term impact ODD has on the lives of children, families, teachers and schools, ${ }^{8}$ as well as the long-term effects until adulthood. ${ }^{9}$ Specifically, these results point to the need to pay attention to the preschool period if the goal is to prevent ODD. On the one hand, preschool age is when the child is acquiring important skills related to ODD, such as self-regulation and executive functioning, and when parents adjust their parenting practices. ${ }^{37}$ It is important to intervene in this period when the early signs of dysfunction become apparent. On the other hand, programmes that 


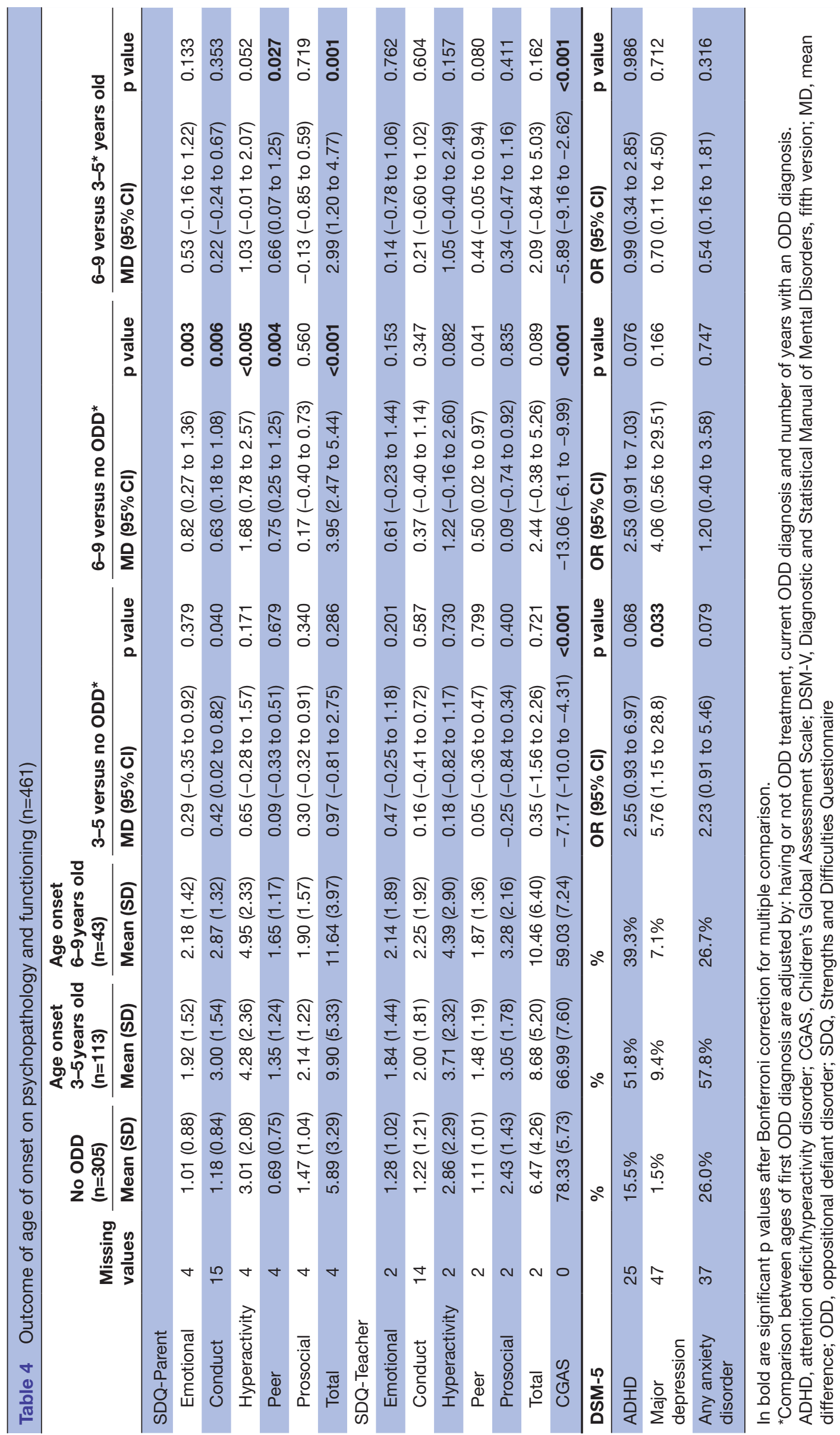


have been shown to effectively treat $\mathrm{ODD}^{38-40}$ and prevent it $^{41} 42$ are currently available.

An early age of onset has typically been associated with worse mental health outcomes. ${ }^{13}$ This is also true for ODD regarding comorbidity. Specifically, the risk of depression in children who debut ODD at preschool age multiplies by 5.76 compared with children without ODD. Comparing early versus later onset after strict control by confounding variables, later onset increases the risk of higher symptomatology (general and in peer problems) and difficulties in functioning. One of the contributions of studying age of onset is to have available information for targeting prevention that focuses on early intervention in incipient mental disorders and on primary prevention of secondary disorders. ${ }^{13}$ Thus, our results once again suggest the need to intervene at early ages. This implication is also supported by the finding that for those starting later (ages 6-9), the impairment in functioning and in symptomatology is more severe. Therefore, paying attention to prodromal indicators and risk factors to prevent the full development of ODD is crucial.

Regarding risk factors, our goal was to confirm the risk of first onset of ODD using some of the main risk factors reported previously in the literature. No previous studies have been carried out with incident cases. The strength of the association for some of the predictors is remarkable. We found that premorbid forms of ODD (subthreshold, high scores in the ODD dimensions irritability and headstrong) were the strongest predictor of onset of full ODD. Identifying premorbid cases is of great value for the indicated prevention of ODD, given that the group at risk presents objective markers (ODD symptoms). Similarly, children with other psychopathology, and specifically ADHD, and individual characteristics, such as difficulties in inhibit-emotional control are also at risk of onset of ODD. Also, our results indicate that difficulties regulating negative emotions are at a higher risk of ODD onset, especially from very early ages, while the risk diminishes with age. Last, unsupportive environments, such as punitive parenting practices and maternal internalising problems, predicted the emergence of an ODD diagnosis, which is also in line with previous literature. ${ }^{154}$ Predictive capability assessed by Harrell's C was generally low to moderate, indicating that to predict first-incident ODD cases, other predictors are needed in addition to the clinical risk factor considered. However, it is necessary to consider the low number of predictors included in each model.

Strengths of the study are that the diagnostic information was obtained via semistructured interviews based on DSM-V criteria, the length of the follow-up period (7 years), the inclusion of two different developmental stages, preschool and childhood, and the fact that the estimates of incidence were not overestimated, given that previous diagnoses until age 3 were also made. Age of onset studies have been carried out mostly through retrospective design, which is a limitation. We studied age of onset through a prospective design. Furthermore, the information on risk factors was obtained from parents and teachers. However, some limitations must be considered when interpreting the results. The diagnostic information, based on data from just one source, the parents and the lower participation of low SES families may have led to bias in the estimates. A second limitation refers to the non-randomness of attrition in 6 out of the 16 outcomes analysed as risk factors of first ODD diagnose. However, as shown in several populations, attrition is associated with adverse psychosocial variables and high levels of psychological distress. ${ }^{45}$ Also, some of the scales of the APQ-Pr presented low internal consistency and the results should thus be interpreted with caution. Finally, as the number of incident cases diminished with age, the statistical power may be limited.

Synthesising, oppositional defiant disorder is one of the most prevalent disorders in our society. It has important consequences in the development of the child and in the functioning of the family. It starts very early in life but we do not know how many new cases appear every year, nor the consequences it has depending on the age of onset. Our study reports that the probability of appearance of oppositional defiant disorders is highest by age 5 and, afterwards, by age 9 , when approaching to puberty. Most of the new cases of oppositional disorder appeared in preschool age $(12.6 \%)$. By age 9 , there is a cumulative risk of new onset of $21.9 \%$. Early onset at preschool age is associated with comorbidity with depression and functional impairment; childhood onset is associated with higher symptomatology and functional impairment. These results indicate the burden of oppositional disorder for public health and point to the need of focusing in preschool age for preventive purposes. To allocate resources in this developmental period and paying attention to prodromal indicators and risk factors to prevent the full development of ODD is crucial. Our results are generalisable to Spanish children mostly from mean and high-mean socioeconomic levels until age 9 .

Acknowledgements We would like to thank the participating schools and families. Contributors LE designed the study and wrote the paper with JBN. JBN analysed data. JMD contributed to data analysis. NdIO and EP collaborated in the writing and editing of the manuscript. All the authors contributed to reviewing the final version of the manuscript.

Funding This work was supported by the Spanish Ministry of Economy and Competitiveness [grants PSI2012-32695 and PSI2015-63965-R (MINECO/FEDER)] and the Secretaria d'Universitats i Recerca, Departament d'Economia i Coneixement of the Generalitat de Catalunya [grant 2014 SGR 312].

Competing interests None declared.

Patient consent for publication Parental/guardian consent obtained.

Ethics approval The authors assert that all procedures contributing to this work comply with the ethical standards of the relevant national and institutional committees on human experimentation and with the Helsinki Declaration of 1975, as revised in 2008, and by the Ethics Committee for Human and Animal Experimentation of the Universitat Autònoma de Barcelona.

Provenance and peer review Not commissioned; externally peer reviewed.

Data sharing statement Data cannot be publicly available due to ethical restrictions protecting the confidentiality of the families involved. Data are available to interested researchers after signing a consent of confidentiality form as the authors had previously signed to obtain the data from the families of the sample. Researchers must be working in clinical child psychology in a publicfunded project. To request the anonymous data, please contact the corresponding author. 
Open access This is an open access article distributed in accordance with the Creative Commons Attribution Non Commercial (CC BY-NC 4.0) license, which permits others to distribute, remix, adapt, build upon this work non-commercially, and license their derivative works on different terms, provided the original work is properly cited, appropriate credit is given, any changes made indicated, and the use is non-commercial. See: http://creativecommons.org/licenses/by-nc/4.0/.

\section{REFERENCES}

1. Polanczyk GV, Salum GA, Sugaya LS, et al. Annual research review: a meta-analysis of the worldwide prevalence of mental disorders in children and adolescents. J Child Psychol Psychiatry 2015;56:345-65.

2. Copeland WE, Wolke D, Shanahan L, et al. Adult functional outcomes of common childhood psychiatric problems: a prospective, longitudinal study. JAMA Psychiatry 2015;72:892-9.

3. La Maison C, Munhoz TN, Santos IS, et al. Prevalence and risk factors of psychiatric disorders in early adolescence: 2004 Pelotas (Brazil) birth cohort. Soc Psychiatry Psychiatr Epidemiol 2018;53:685-97.

4. Nock MK, Kazdin AE, Hiripi E, et al. Lifetime prevalence, correlates, and persistence of oppositional defiant disorder: results from the National Comorbidity Survey Replication. J Child Psychol Psychiatry 2007;48:703-13.

5. Drabick DA, Ollendick TH, Bubier JL. Co-occurrence of ODD and anxiety: shared risk processes and evidence for a dual-pathway model. Clin Psychol 2010;17:307-18.

6. Mikolajewski AJ, Taylor J, lacono WG. Oppositional defiant disorder dimensions: genetic influences and risk for later psychopathology. $J$ Child Psychol Psychiatry 2017;58:702-10.

7. Boekamp JR, Liu RT, Martin SE, et al. Predictors of partial hospital readmission for young children with oppositional defiant disorder. Child Psychiatry Hum Dev 2018;49:505-11.

8. Burke JD, Rowe R, Boylan K. Functional outcomes of child and adolescent oppositional defiant disorder symptoms in young adult men. J Child Psychol Psychiatry 2014;55:264-72.

9. Leadbeater BJ, Ames ME. The Longitudinal Effects of Oppositional Defiant Disorder Symptoms on Academic and Occupational Functioning in the Transition to Young Adulthood. J Abnorm Child Psychol 2017;45:749-63.

10. Greenland S, Rothman KJ. Measures of occurrence. In: Rothman KJ Greenland S, Lash TL, eds. Modern epidemiology. Philadelphia, PA: Lippincott Williams \& Wilkins, 2008:32-50.

11. Roberts RE, Roberts CR, Chan W. One-year incidence of psychiatric disorders and associated risk factors among adolescents in the community. J Child Psychol Psychiatry 2009;50:405-15.

12. Benjet C, Borges G, Méndez E, et al. Eight-year incidence of psychiatric disorders and service use from adolescence to early adulthood: longitudinal follow-up of the Mexican Adolescent Mental Health Survey. Eur Child Adolesc Psychiatry 2016;25:163-73.

13. Kessler RC, Amminger GP, Aguilar-Gaxiola S, et al. Age of onset of mental disorders: a review of recent literature. Curr Opin Psychiatry 2007;20:359-64.

14. de Girolamo G, Dagani J, Purcell R, et al. Age of onset of mental disorders and use of mental health services: needs, opportunities and obstacles. Epidemiol Psychiatr Sci 2012;21:47-57.

15. Lavigne JV, Herzing LB, Cook EH, et al. Gene $\times$ environment effects of serotonin transporter, dopamine receptor D4, and monoamine oxidase A genes with contextual and parenting risk factors on symptoms of oppositional defiant disorder, anxiety, and depression in a community sample of 4-year-old children. Dev Psychopathol 2013;25:555-75.

16. Wichstrøm L, Penelo E, Rensvik Viddal K, et al. Explaining the relationship between temperament and symptoms of psychiatric disorders from preschool to middle childhood: hybrid fixed and random effects models of Norwegian and Spanish children. $J$ Child Psychol Psychiatry 2018;59:285-95.

17. Matthys W, Vanderschuren LJ, Schutter DJ, et al. Impaired neurocognitive functions affect social learning processes in oppositional defiant disorder and conduct disorder: implications for interventions. Clin Child Fam Psychol Rev 2012;15:234-46.

18. Demmer DH, Hooley M, Sheen J, et al. Sex differences in the prevalence of oppositional defiant disorder during middle childhood: a meta-analysis. J Abnorm Child Psychol 2017;45:313-25.

19. Harvey EA, Breaux RP, Lugo-Candelas Cl. Early development of comorbidity between symptoms of attention-deficit/hyperactivity disorder (ADHD) and oppositional defiant disorder (ODD). J Abnorm Psychol 2016;125:154-67.

20. Rowe R, Costello EJ, Angold A, et al. Developmental pathways in oppositional defiant disorder and conduct disorder. J Abnorm Psychol 2010;119:726-38.

21. de Graaf R, ten Have M, Tuithof M, et al. First-incidence of DSM-IV mood, anxiety and substance use disorders and its determinants: results from the Netherlands Mental Health Survey and Incidence Study-2. J Affect Disord 2013;149(1-3):100-7.

22. Stringaris A, Goodman R. Three dimensions of oppositionality in youth. J Child Psychol Psychiatry 2009;50:216-23.

23. Shankman SA, Lewinsohn PM, Klein DN, et al. Subthreshold conditions as precursors for full syndrome disorders: a 15-year longitudinal study of multiple diagnostic classes. J Child Psychol Psychiatry 2009;50:1485-94.

24. Roberts RE, Fisher PW, Turner JB, et al. Estimating the burden of psychiatric disorders in adolescence: the impact of subthreshold disorders. Soc Psychiatry Psychiatr Epidemiol 2015;50:397-406.

25. Ezpeleta L, de la Osa N, Doménech JM. Prevalence of DSM-IV disorders, comorbidity and impairment in 3-year-old Spanish preschoolers. Soc Psychiatry Psychiatr Epidemiol 2014;49:145-55.

26. Goodman R. Psychometric properties of the strengths and difficulties questionnaire. J Am Acad Child Adolesc Psychiatry 2001;40:1337-45.

27. Ezpeleta L, de la Osa N, Granero R, et al. The Diagnostic Interview of Children and Adolescents for Parents of Preschool and Young Children: psychometric properties in the general population. Psychiatry Res 2011;190:137-44.

28. Shaffer D, Gould MS, Brasic J, et al. A children's global assessment scale (CGAS). Arch Gen Psychiatry 1983;40:1228-31.

29. Putnam SP, Rothbart MK. Development of Short and Very Short Forms of the Children's Behavior Questionnaire. J Pers Assess 2006;87:102-12.

30. Gioia GA, Espy KA, Isquith PK. Behavior rating inventory of executive function - preschool version. Lutz, FL: Psychological Assessment Resources, 2003.

31. Frick PJ. Alabama parenting questionnaire, 1991.

32. de la Osa N, Granero R, Penelo E, et al. Psychometric properties of the Alabama Parenting Questionnaire - Preschool revision (APQ$\mathrm{Pr}$ ) in 3 year-old Spanish preschoolers. Journal of Child and Family Studies 2014;23:776-84

33. Achenbach TM, Rescorla LA. Manual for the ASEBA adult forms \& profiles. Burlington, VT: University of Vermont, Research Center for Children, Youth \& Families, 2003.

34. Kaplan EL, Meier P. Nonparametric Estimation from Incomplete Observations. J Am Stat Assoc 1958;53:457-81.

35. Lin DY, Wei LJ. The Robust inference for the cox proportional hazards model. J Am Stat Assoc 1989;84:1074-8.

36. Newson RB. Comparing the Predictive Powers of Survival Models Using Harrell's C or Somers' D. Stata J 2010;10:339-58.

37. Eisenberg N. Emotion, regulation, and moral development. Annu Rev Psychol 2000;51:665-97.

38. Fossum S, Handegård BH, Adolfsen $\mathrm{F}$, et al. A meta-analysis of longterm outpatient treatment effects for children and adolescents with conduct problems. J Child Fam Stud 2016;25:15-29.

39. Hobbel S, Drugli MB. Symptom changes of oppositional defiant disorder after treatment with the Incredible Years Program. Nord $J$ Psychiatry 2013;67:97-103.

40. Kaminski JW, Claussen AH. Evidence base update for psychosocial treatments for disruptive behaviors in children. $J$ Clin Child Adolesc Psychol 2017; 46:477-99.

41. Muratori P, Bertacchi I, Giuli C, et al. Coping Power Adapted as Universal Prevention Program: Mid Term Effects on Children's Behavioral Difficulties and Academic Grades. J Prim Prev 2016;37:389-401.

42. Winther J, Carlsson A, Vance A. A pilot study of a school-based prevention and early intervention program to reduce oppositional defiant disorder/conduct disorder. Early Interv Psychiatry 2014;8:181-9.

43. Tung I, Lee SS. Negative parenting behavior and childhood oppositional defiant disorder: differential moderation by positive and negative peer regard. Aggress Behav 2014;40:79-90.

44. Fischer EH, Dornelas EA, Goethe JW. Characteristics of people lost to attrition in psychiatric follow-up studies. J Nerv Ment Dis 2001;189:49-55.

45. Granero R, Ezpeleta L, Doménech JM. Features associated with non-participation and abandonment in mental health epidemiological designs of socially-at-risk children and adolescents. Soc Psychiatry Psychiatr Epidemiol 2007;42:251-8. 